\title{
Multidetector CT Angiography for the Detection of Colonic Diverticular Bleeding: When, How, and Why?
}

\author{
Romaric Loffroy
}

Received: 25 April 2013/Accepted: 26 April 2013/Published online: 22 May 2013

(c) Springer Science+Business Media New York 2013

Acute lower GI bleeding is responsible for 1-2 \% of hospital emergencies, $15 \%$ of which manifest as massive, lifethreatening bleeding. Initial clinical management focuses on identifying the site and cause of bleeding using scintigraphy, arteriography, and colonoscopy [1-3]. Although optical colonoscopy is the procedure of choice, its use poses challenges in the emergency setting [1]. Administration of bowel preparation, not always feasible in cases of massive bleeding, can delay the procedure for several hours; the presence of clots, blood, and stool in patients without preparation may hinder the visualization of the bleeding point, increasing the rate of incomplete studies when compared with nonemergent examinations. Furthermore, in 10-12\% of cases, the source of bleeding may be extracolonic [4]. Angiography is an invasive procedure usually reserved for therapeutic purposes in patients with more severe, life-threatening bleeding. Technetium $99^{\mathrm{m}}$ labeled red blood cell scintigraphy is more sensitive than arteriography for identifying lower rate bleeding, although its emergency availability is variable, limiting its usefulness [4]. Computed tomography (CT) and, more specifically, CT enterography is commonly used as a second-line procedure in nonemergent patients with unexplained GI bleeding after negative upper and lower endoscopy examinations [5]. Since CT is rapid, noninvasive, and highly reproducible, it is commonly used in the acute setting for evaluating patients with lower GI bleeding emergencies, although optimal timing and patient characteristics for the diagnosis of colonic diverticular bleeding have not been

\section{R. Loffroy $(\bowtie)$}

Department of Vascular and Interventional Radiology, Bocage

Teaching Hospital, University of Burgundy School of Medicine, Dijon, France

e-mail: romaric.loffroy@ chu-dijon.fr established. In this issue, Obana et al. [6] prospectively explored the utility of contrast-enhanced CT in 52 patients with colonic diverticular bleeding testing the hypothesis that CT complements colonoscopy in this setting. Favorable patient characteristics, based on univariate analysis, included feasibility of examination within $2 \mathrm{~h}$ of the last hematochezia, re-bleeding in hospital, or past history of diverticular bleeding.

Detection and localization of the bleeding source enable successful catheter-directed endovascular therapy. Although emergent colonoscopy remains the primary investigation in lower GI bleeding, it is often negative or impractical during the acute phase in hemodynamically unstable patients, as confirmed in the accompanying study [6]. In many centers, CT angiography is performed if endoscopy fails to identify the bleeding source with conventional mesenteric angiography reserved for patients with CT evidence of active contrast extravasatio or hemodynamic instability due to massive hemorrhage. CT may visualize acute lower GI bleeding at a bleeding rate less than the $0.5 \mathrm{ml} / \mathrm{min}$ limit cited for mesenteric angiography, perhaps as low as $0.2 \mathrm{ml} / \mathrm{min}$ [5]. The additional information provided by $\mathrm{CT}$ before attempted therapeutic angiographic procedures improves the preoperative localization and characterization of the bleeding source, expediting the selective catheterization of bleeding vessels, thereby significantly facilitating embolization.

Relevant findings evaluated in this study were the presence and location of active or recent bleeding and the identification of the potential bleeding lesion [6]. Active bleeding from colonic diverticula was identified in only $15.4 \%(8 / 52)$ of the patients included in the study, lower than in other series that included only massive or severe bleeding, probably because most patients were hemodynamically stable at the time CT was performed. In many 
Table 1 Key points

\begin{tabular}{|c|c|}
\hline $\begin{array}{l}\text { Key } \\
\text { point }\end{array}$ & Description \\
\hline 1 & Acute colonic diverticular bleeding represents a significant challenge for clinicians \\
\hline 2 & Endoscopy still plays the primary role in the initial investigation of lower GI bleeding \\
\hline 3 & $\begin{array}{l}\text { However, endoscopy may be negative or impractical in some patients. It is this subgroup of patients in whom CT may make the } \\
\text { largest impact }\end{array}$ \\
\hline 4 & $\begin{array}{l}\text { There is increasing data to suggest that CT should be the "next step" investigative procedure in cases of active lower GI hemorrhage } \\
\text { and should supersede more invasive and time-consuming procedures such as catheter angiography and red cell scintigraphy } \\
\text { respectively }\end{array}$ \\
\hline 5 & $\begin{array}{l}\text { In cases of colonic diverticular bleeding, CT provides a rapid, noninvasive method of accurately localizing the site of bleeding, } \\
\text { facilitating improved triaging of patients to lesion-directed catheter-based angiography or open surgery to treat the bleeding process } \\
\text { as appropriate }\end{array}$ \\
\hline 6 & $\begin{array}{l}\text { In some centers, the additional information provided by } \mathrm{CT} \text { prior to attempts at therapeutic angiographic procedures has led to faster } \\
\text { selective catheterization of bleeding vessels, shorter procedure times, decreased volumes of intravenous contrast being used and has } \\
\text { led to decreased patient and operator radiation exposure within the angiography suite }\end{array}$ \\
\hline
\end{tabular}

cases, acute GI bleeding occurs intermittently or ceases spontaneously, presenting a major diagnostic and treatment dilemma [7]. Since the rate of even massive acute GI bleeding can vary from minute to minute, failure to demonstrate active bleeding may therefore not indicate cessation of bleeding in all cases. Delayed follow-up examinations may be required to localize the bleeding. Therefore, to maximize detection capabilities, it is crucial that CT angiography should be performed as soon as possible while the patient is actively bleeding [7, 8]. Indeed, the likelihood of identifying the bleeding source is higher when CT scan is carried out in hemodynamically unstable patients, with monitoring provided by intensive care unit physicians.

Factors influencing the ability to visualize active bleeding at CT are multiple, as previously described, and include the nature of the bleeding lesion (bleeding rate, intermittence), patient factors (hemodynamic status, body mass index), but also the CT technique (rate of injection, concentration of iodine in contrast material, number of phases, type of scanner, post-processing), and the experience of the radiologist [8, 9]. The addition of imaging phases to the CT angiography study may provide more information but also increases the total radiation dose. The highest sensitivity for detecting intestinal bleeding is achieved by means of a dual-phase protocol (arterial and portal venous phases) which improves detection of extravasated contrast medium during the arterial phase and also provides information about the cause of the bleeding $[4,8]$, a technique not used in the accompanying study.

Some of the limitations of the accompanying study include a small total patient number, probably due to the authors limiting the study to patients for whom an emergent colonoscopy was requested by the admitting emergency physician. This limitation was imposed in order to increase the proportion of patients who would be predicted to have CT angiography. Also, there was not a true control group because of the difficulty to set up, which is another limitation of the study. Rather than using the severity of the hemorrhagic episode as a criterion for study inclusion, the authors considered all patients who required colonoscopy. Other limitations of CT angiography for this clinical application include the lack of therapeutic options, the use of ionizing radiation, and risks associated with intravenous contrast media.

In summary, the use of multidetector CT angiography in the diagnostic work-up of patients with acute GI bleeding continues to increase due to improvements in scanning technology [4, 8]. CT angiography is a noninvasive, rapid, reproducible, and widely available technique that can be performed successfully in the majority of patients presenting with an acute episode of acute lower GI bleeding from colonic diverticula, without the need for colonic preparation. Positive contrast-enhanced multidetector CT can define with a high degree of accuracy the location and at times the cause of active lower GI bleeding, which combined with anatomic information can effectively inform subsequent angiography or surgery. Multiphasic imaging enables direct visualization of active bleeding into the bowel [4, 5]. Due to the intermittent nature of GI bleeding, CT angiography should be performed as soon as possible after the clinical detection of active bleeding to maximize detection potential. Nevertheless, lesions can be identified even if an active bleeding site is not [8]. Rather than restricting it to cases where colonoscopy fails initially, CT angiography is proposed as a first diagnostic modality in the evaluation of patients with substantial bleeding (Table 1). 


\section{References}

1. Green BT, Rockey DC, Portwood G, et al. Urgent colonoscopy for evaluation and management of acute lower gastrointestinal hemorrhage: a randomized controlled trial. Am J Gastroenterol. 2005; 100:2395-2402.

2. Zink SI, Ohki SK, Stein B, et al. Noninvasive evaluation of active lower gastrointestinal bleeding: comparison between contrastenhancedMDCT and 99mTc-labeled RBC scintigraphy. AJR Am J Roentgenol. 2008;191:1107-1114.

3. Millward SF. ACR appropriateness criteria on treatment of acute nonvariceal gastrointestinal tract bleeding. J Am Coll Radiol. 2008;5:550-554.

4. Marti M, Artigas JM, Garzon G, Alvarez-Sala R, Soto JA. Acute lower intestinal bleeding: feasibility and diagnostic performance of CT angiography. Radiology. 2012;262:109-116.

5. Laing CJ, Tobias T, Rosenblum DI, Banker WL, Tseng L, Tamarkin SW. Acute gastrointestinal bleeding: emerging role of multi detector CT angiography and review of current imaging techniques. Radiographics. 2007;27:1055-1070.

6. Obana T, Fujita N, Sugita R, et al. Prospective evaluation of contrast-enhanced computed tomography for the detection of colonic diverticular bleeding. Dig Dis Sci. (Epub ahead of print). doi:10.1007/s10620-013-2629-6.

7. Loffroy R, Guiu B, D'Athis P, et al. Arterial embolotherapy for endoscopically unmanageable acute gastroduodenal hemorrhage: predictors of early rebleeding. Clin Gastroenterol Hepatol. 2009;7:515-523.

8. Scheffel H, Pfammatter T, Wildi S, Bauerfeind P, Marincek B, Alkadhi H. Acute gastrointestinal bleeding: detection of source and etiology with multi-detector-row CT. Eur Radiol. 2007;17: $1555-1565$.

9. Loffroy R, Cercueil JP, Guiu B, Krause D. Detection and localization of acute lower gastrointestinal bleeding prior to therapeutic endovascular embolization: a challenge! Am J Gastroenterol. 2009;104:3108-3109. 